\title{
The four dimensional site-diluted Ising model: a finite-size scaling study
}

\author{
H. G. Ballesteros*, L. A. Fernández ${ }^{\dagger}$, \\ V. Martín-Mayor ${ }^{\ddagger}$, A. Muñoz Sudupe ${ }^{\S}$, \\ Departamento de Física Teórica I, Facultad de CC. Físicas, \\ Universidad Complutense de Madrid, 28040 Madrid, Spain. \\ G. Parisi and J. J. Ruiz-Lorenzo".
Dipartimento di Fisica and Istituto Nazionale di Fisica Nucleare,
Università di Roma "La Sapienza", P. A. Moro 2, 00185 Roma, Italy.
}

July 16, 1997

\begin{abstract}
Using finite-size scaling techniques, we study the critical properties of the site-diluted Ising model in four dimensions. We carry out a high statistics Monte Carlo simulation for several values of the dilution. The results support the perturbative scenario: there is only the Ising fixed point with large logarithmic scaling corrections. We obtain, using the Perturbative Renormalization Group, functional forms for the scaling of several observables that are in agreement with the numerical data.
\end{abstract}

Key words: Lattice. Monte Carlo. Disordered Systems. Critical exponents. Finite size scaling. $\epsilon$-expansion.

PACS: 05.50.+q;05.70.Jk;75.10.Nr;75.40.Mg

\footnotetext{
*hector@lattice.fis.ucm.es

$\dagger$ laf@lattice.fis.ucm.es

$\ddagger$ victor@lattice.fis.ucm.es

$\S$ sudupe@lattice.fis.ucm.es

Tgiorgio.parisi@roma1.infn.it

$\|_{\text {ruiz@chimera.roma1.infn.it }}$
} 


\section{Introduction}

A possible way to obtain new Universality Classes (UC) is to add disorder to known pure systems. The Harris criterion [1] says that, if the specific heat diverges with a power law in the pure system, then, the disorder will change the critical behavior of the model, i.e. a new UC will appear. Conversely, if the specific heat does not diverge in the pure system, then, the critical exponents of the disordered system will remain unchanged. In the limiting case, what amounts to a discontinuous or a logarithmically divergent specific heat, the criterion does not apply and we need to study analytically or/and numerically the system.

The quest and the characterization of new UC are very important in dimensions two and three (with a direct relevance on Condensed Matter Physics) and in four dimensions (with implications on High Energy Physics). In the last case it is crucial to characterize all the possible $\mathrm{UC}$, in order to be able to define a Field Theory on a non-perturbative basis. As the Gaussian model gives a trivial one (i.e. at long distances the theory will be free) we are interested in finding a non-Gaussian UC.

In this paper we will study the four dimensional site-diluted Ising model that was previously studied [2] by two of the authors, who calculated numerically the critical exponents, analyzing the divergences with the temperature. Their results pointed to non Gaussian critical exponents, for large values of the dilution, but it was noted the possibility of a crossover between the behavior found and the Gaussian one.

In order to obtain accurate measures of the critical properties we have repeated the simulations in a greater number of spin configurations. The use of Finite Size Scaling (FSS) techniques allows us to work in large lattices at the critical point.

Using the Perturbative Renormalization Group (PRG) equations we calculate the dependence of the observables at the critical point with the scale of the system including logarithmic corrections. These are different from the pure system.

Our determination of the critical exponents and other critical properties matches very well with the predictions of the PRG: a Gaussian critical behavior with logarithmic corrections. We check this behavior along the critical line in a wide range of concentrations, from $p=0.8$ to $p=0.3$ (the percolation threshold is near 0.2). We remark that a scenario based on hyperscaling seems completely unlikely from our numerical simulations. 
The structure of the paper is as follows. In the next section we define the model and the observables. In section 3 we present our analytical calculations, obtaining the FSS formulas and calculating the values of two different Binder cumulants in the thermodynamic limit. In section 4 we describe the numerical methods and the different techniques that we will use to analyze the observables. In section 5 we show our numerical results confronting them with the analytical predictions. Finally we report the conclusions.

\section{The model}

The model we study numerically in this article, is defined in terms of $\mathrm{Z}_{2}$ spin-variables placed in the nodes of a hypercubic four-dimensional lattice. The action is

$$
S=-\beta \sum_{<i, j>} \epsilon_{i} \epsilon_{j} \sigma_{i} \sigma_{j}
$$

where the sum is extended over nearest-neighbors, and the $\epsilon_{i}$ are quenched, uncorrelated random variables, taking the value 1 , with probability $p$, and 0 with probability $1-p$. An actual $\left\{\epsilon_{i}\right\}$ configuration, will be called a sample from now on. For every observable it is understood that first one performs the Ising model calculation and then the $\epsilon$-average.

In the following, we shall denote an Ising average with brackets, while the sample average will be overlined. The observables will be denoted with calligraphic letters, i.e. $\mathcal{O}$ and with italics the double average $O=\overline{\langle\mathcal{O}\rangle}$. We define the total nearest-neighbor energy and the normalized magnetization as

$$
\mathcal{E}=\sum_{\langle i, j\rangle} \epsilon_{i} \sigma_{i} \epsilon_{j} \sigma_{j}, \quad \mathcal{M}=\frac{1}{V} \sum_{i} \epsilon_{i} \sigma_{i},
$$

$V$ being the volume (defined as $L^{4}$, where $L$ is the lattice size). We also define the susceptibility as

$$
\chi=V \overline{\left\langle\mathcal{M}^{2}\right\rangle} .
$$

Another very useful quantity is the Binder parameter: 


$$
g_{4}=\frac{3}{2}-\frac{1}{2} \frac{\overline{\left\langle\mathcal{M}^{4}\right\rangle}}{\overline{\left\langle\mathcal{M}^{2}\right\rangle^{2}}} .
$$

Other kind of Binder parameter, meaningless for the pure system, can be defined as

$$
g_{2}=\frac{\overline{\left\langle\mathcal{M}^{2}\right\rangle^{2}-{\overline{\left\langle\mathcal{M}^{2}\right.}}^{2}}}{{\overline{\left\langle\mathcal{M}^{2}\right\rangle}}^{2}} .
$$

A very convenient definition of the correlation length in a finite lattice, reads [3]

$$
\xi=\left(\frac{\chi / F-1}{4 \sin ^{2}(\pi / L)}\right)^{\frac{1}{2}},
$$

where $F$ is defined in terms of the Fourier transform of the magnetization

$$
\mathcal{F}(\boldsymbol{k})=\frac{1}{V} \sum_{\boldsymbol{r}} \mathrm{e}^{\mathrm{i} \boldsymbol{k} \cdot \boldsymbol{r}} \epsilon_{\boldsymbol{r}} \sigma_{\boldsymbol{r}}
$$

as

$$
F=\frac{V}{4} \overline{\left\langle|\mathcal{F}(2 \pi / L, 0,0,0)|^{2}+\text { permutations }\right\rangle} .
$$

This definition is very well behaved for the finite-size scaling (FSS) method we employ [4], and it is also fair natural for considerations about triviality [5]. Finally, we measure the specific heat

$$
C=V^{-1} \overline{\left\langle\mathcal{E}^{2}\right\rangle-\langle\mathcal{E}\rangle^{2}}
$$

\section{Analytical predictions}

The field-theoretical study of the model (1) is performed by means of a $\phi^{4}$ theory with a random mass term, whose action is

$$
S[\phi]=\int \mathrm{d}^{d} x\left(\frac{1}{2}\left(\partial_{\mu} \phi\right)^{2}+\frac{1}{2} m^{2}(x) \phi^{2}+\frac{1}{4 !} v \phi^{4}\right) .
$$

Here it is assumed that the mass term is a quenched, spatially-uncorrelated, stochastic variable. We will later argue that the only relevant parameters of the disorder distribution are its mean, $r$, and variance, $\Delta^{2}$, so we will assume for simplicity the Gaussian distribution 


$$
\mathrm{d} P\left[m^{2}(x)\right] \propto \mathrm{d}\left[m^{2}\right] \exp \left(-\frac{\left(m^{2}(x)-r\right)^{2}}{2 \Delta^{2}}\right) .
$$

When the disorder is quenched we need to compute, in a first step, the free energy of the system for a given choice of the disorder (in this case of the mass term), and then average this free energy with the probability distribution of the disorder. To manage this kind of problems it is very useful to use the so-called replica trick [6].

Let us introduce $n$ replicas of the initial system, $\phi_{i}$, with $i=1, \ldots, n$. The average of the replicated partition function over the Gaussian disorder will be denoted by overlines.

$$
F=\overline{\log \mathcal{Z}}=\lim _{n \rightarrow 0} \frac{1}{n}\left(\overline{\mathcal{Z}^{n}}-1\right) .
$$

Now we can define an effective action by means of

$$
\overline{\mathcal{Z}^{n}}=\mathcal{Z}_{\text {eff }}=\int \mathrm{d}\left[\phi_{i}\right] \exp \left(-S_{\text {eff }}\left[\phi_{i}\right]\right)
$$

with

$$
S_{\text {eff }}\left[\phi_{i}\right]=\int \mathrm{d}^{d} x\left[\frac{1}{2} \sum_{i=1}^{n}\left(\partial_{\mu} \phi_{i}\right)^{2}+\frac{r}{2} \sum_{i=1}^{n} \phi_{i}^{2}+\frac{u}{4 !}\left[\sum_{i=1}^{n} \phi_{i}^{2}\right]^{2}+\frac{v}{4 !} \sum_{i=1}^{n} \phi_{i}^{4}\right]
$$

where $u=-3 \Delta^{2}$. This gives us a starting point for the analytical calculation. The $n \rightarrow 0$ limit should be taken at the end.

For $v=0$ the action is $O(n)$-invariant. When $u=0$ the action describes $n$ decoupled Ising models. We remark that $u$ is negative and proportional to the dilution. It is possible to show that for a non-Gaussian distribution, terms associated with higher connected momenta of the distribution appear in the effective action. The $s$-momentum couples to $\phi^{2 s}$ thus, if $s>2$, is irrelevant in four dimensions and can be neglected. In our numerical simulation a site is occupied with probability $p$, so $\Delta^{2}=p(1-p)$.

The action (14) was studied in ref. [7] by using PRG techniques. Considering a differential dilatation, the following equations are obtained:

$$
\frac{\mathrm{d} r}{\mathrm{~d} \log b}=2 r+4 K_{d}(2 u+3 v)(1-r)
$$




$$
\begin{aligned}
& \frac{\mathrm{d} v}{\mathrm{~d} \log b}=\epsilon v-12 K_{d} v(4 u+3 v), \\
& \frac{\mathrm{d} u}{\mathrm{~d} \log b}=\epsilon u-8 K_{d} u(4 u+3 v),
\end{aligned}
$$

where $\epsilon \equiv 4-d, d$ being the dimension, $K_{d}$ is a constant that depends on the dimension, and $b$ is the Renormalization Group (RG) scaling factor. In the previous formulas third order terms in $u, v$ have been neglected, and the $n \rightarrow 0$ limit has been considered. In the following we shall set $\epsilon=0$, as we study a four dimensional problem.

The initial conditions of the system, typically verify $\left|u_{0}\right| \ll v_{0}$. Therefore, the RG evolution, driven by eqs. (15), will present two interesting regimes

1. A transient regime in which $-u \sim v^{2 / 3}$. We find $v(b) \sim 1 / \log b$ or equivalently $v(L) \sim 1 / \log L$.

2. An asymptotic regime reached by following the $\mathrm{RG}$ evolution until the equation $4 u+3 v=O\left(u^{2}\right)$ finally holds. We obtain, including the next term in the perturbative expansion, $u^{2}(b), v^{2}(b) \propto 1 / \log b$, or $1 / \log L$.

Thus, we expect a crossover between the pure situation where the relevant coupling, $v$, goes to zero as $v(L) \sim 1 / \log L$ and the disordered one where $u$ and $v$ are similar in magnitude and $v(L) \sim 1 / \sqrt{\log L}$, i.e. the relevant coupling $(u$ or $v)$ goes to zero more slowly than in the initial regime.

Defining $t \equiv r-4 K_{4} u$, eqs. (15) in the asymptotic regime reduce to

$$
\begin{aligned}
& \frac{\mathrm{d} t}{\mathrm{~d} \log b}=2 t+8 K_{4} u t, \\
& \frac{\mathrm{d} u}{\mathrm{~d} \log b}=-\frac{1696}{3} K_{4}^{2} u^{3} .
\end{aligned}
$$

For large $b$, the solutions are

$$
\begin{aligned}
& t(b)=t_{0} b^{2} \exp \left[-2 \sqrt{\frac{3 \log b}{53}}\right], \\
& u(b)=-\sqrt{\frac{3}{3392 K_{4}^{2} \log b}},
\end{aligned}
$$


where $t_{0}$ is an integration constant and in the large $b$ regime $u(b)$ does not depend on the initial condition $u_{0}$.

Using these formulas it is possible to obtain the expressions for the correlation length, susceptibility and specific heat as functions of the reduced temperature.

We find just one slight difference with ref. [7]. The equation for the wave function renormalization, $\zeta(b)$, is

$$
\frac{\mathrm{d} \zeta}{\mathrm{d} \log b}=-\gamma_{\phi}(u, v)=-8 K_{4}^{2}\left(2 u^{2}+6 u v+3 v^{2}\right) .
$$

In the pure model $\zeta$ is constant, but in the asymptotic region one obtains $\zeta(b) \propto(\log b)^{1 / 212}$ which affects to the susceptibility. This correction is negligible from a practical point of view, but it could be important in related models (for instance in spin glasses and percolation [8]). So the formula for the susceptibility reads:

$$
\chi \simeq t_{0}^{-1} \exp \left[\sqrt{\frac{6}{53}}\left|\log t_{0}\right|^{1 / 2}\right]\left|\log t_{0}\right|^{1 / 106},
$$

where we have used that $\chi=\zeta^{2} \xi^{2}$ [9]. In ref. [7] the term $\zeta^{2}$ is absent.

\subsection{Calculation of Binder Cumulants}

In this section we will calculate the values of $g_{2}$ and $g_{4}$, with the techniques introduced in ref. [10] for the study of finite geometries using Field Theoretical methods.

The main idea is to expand the field $\phi(x)$ in Fourier modes. In a finite geometry the biggest contribution comes from the zero mode. It can be shown that it has to be treated non perturbatively while this is not necessary for the rest of the modes [10].

In our case the effective action for the zero mode, that we will denote as $\psi_{i}$ is, in a $L^{d}$ volume and just at the MF critical point (i.e. $r=0$ ),

$$
S_{\mathrm{eff}}\left[\psi_{i}\right]=L^{d}\left[\frac{u}{4 !}\left(\sum_{i=1}^{n} \psi_{i}^{2}\right)^{2}+\frac{v}{4 !} \sum_{i=1}^{n} \psi_{i}^{4}\right] .
$$

and the partition function is

$$
\mathcal{Z}_{\text {eff }}(n)=\int\left(\prod_{i=1}^{n} \mathrm{~d} \psi_{i}\right) \exp \left(-S_{\text {eff }}\left[\psi_{i}\right]\right) .
$$


In the asymptotic regime, the relation $4 u+3 v \simeq 0$ is satisfied with good precision, and so:

$$
\mathcal{Z}_{\text {eff }}(n)=\frac{1}{\sqrt{3 \pi}} \int\left(\prod_{i=1}^{n} \mathrm{~d} \psi_{i}\right) \mathrm{d} \lambda \exp \left[-\frac{1}{3} \lambda^{2}+\lambda \sum_{i} \psi_{i}^{2}-\sum_{i} \psi_{i}^{4}\right]
$$

where we have introduced a Gaussian $\lambda$-integration in order to decouple the term $\left(\sum_{i=1}^{n} \psi_{i}^{2}\right)^{2}$. It is possible to see that dimensionless ratios, like $g_{4}$ and $g_{2}$, do not depend on the specific value of $v$, thereby we have also fixed $v=4 ! / L^{d}$ in the previous formula and in the rest of the section.

We remark that the ratio between $u$ and $v$ in $d \leq 4$ is universal, because there, we have a (limiting) fixed ratio $u / v$. For instance, in four dimensions $u$ and $v$ go to zero with a limiting ratio, $u / v \rightarrow-4 / 3$, whereas in $d<4, u$ and $v$ go to the non trivial fixed points $u^{*}$ and $v^{*}$, respectively, and $u / v \rightarrow u^{*} / v^{*}$. In $d>4$ it is impossible to fix this ratio: it depends on the parameters in the Hamiltonian. It is possible to show that using the $\epsilon$-expansion one can obtain for a Binder cumulant the MF value (calculated in $d>4$ ) plus corrections that are proportional to real and positive powers of $\epsilon$ [10. So we can fix the ratio between $u$ and $v$ to the four dimensions value and then do the computation directly in $d>4$ (i.e. avoiding the loop effects).

We can perform the integrals on the $\psi$ variables

$$
\mathcal{Z}_{\text {eff }}(n)=\frac{1}{\sqrt{3 \pi}} \int \mathrm{d} \lambda \mathrm{e}^{-\lambda^{2} / 3} I_{0}(\lambda)^{n},
$$

where

$$
I_{m}(\lambda) \equiv \int \mathrm{d} \psi \exp \left[\lambda \psi^{2}-\psi^{4}\right] \psi^{m} .
$$

For the calculation of the cumulants we need to evaluate with the action (22) the following averages [6]:

$$
\begin{aligned}
\overline{\left\langle\mathcal{M}^{2}\right\rangle} & \rightarrow\left\langle\psi_{a}^{2}\right\rangle, \\
\frac{\left\langle\mathcal{M}^{4}\right\rangle}{\left\langle\mathcal{M}^{2}\right\rangle^{2}} & \rightarrow\left\langle\psi_{a}^{4}\right\rangle,
\end{aligned}
$$

For instance

$$
\left\langle\psi_{a}^{2 m}\right\rangle=\left(\sqrt{3 \pi} \mathcal{Z}_{\text {eff }}\right)^{-1} \int \mathrm{d} \lambda e^{-\lambda^{2} / 3} I_{2 m}(\lambda) I_{0}(\lambda)^{n-1} .
$$


The moments in the $n \rightarrow 0$ limit are

$$
\begin{aligned}
\left\langle\psi_{a}^{2 m}\right\rangle & =\frac{1}{\sqrt{3 \pi}} \int \mathrm{d} \lambda \frac{I_{2 m}(\lambda)}{I_{0}(\lambda)} \mathrm{e}^{-\lambda^{2} / 3}, \\
\left\langle\psi_{a}^{2} \psi_{b}^{2}\right\rangle & =\frac{1}{\sqrt{3 \pi}} \int \mathrm{d} \lambda\left[\frac{I_{2}(\lambda)}{I_{0}(\lambda)}\right]^{2} \mathrm{e}^{-\lambda^{2} / 3},
\end{aligned}
$$

where $a \neq b$.

Evaluating numerically the previous integrals we obtain

$$
\begin{array}{ll}
g_{4}^{\text {disordered }} & =0.32455 \ldots, \\
g_{2}^{\text {disordered }} & =0.31024 \ldots .
\end{array}
$$

We recall that the MF values for the moments in the pure case are [10]

$$
\left\langle\mathcal{M}^{2 m}\right\rangle=\frac{I_{2 m}(0)}{I_{0}(0)},
$$

and for the cumulants

$$
\begin{aligned}
& g_{4}^{\text {pure }}=0.40578 \ldots, \\
& g_{2}^{\text {pure }}=0 .
\end{aligned}
$$

Now the interpretation of the formulas (27) is clear. We have a $\lambda$-model with action $S=\lambda \psi^{2}-\psi^{4}$, with $\lambda$ distributed with a Gaussian weight $\exp \left(-\lambda^{2} / 3\right)$. Averaging with this probability distribution the moments $I_{2 m}(\lambda) / I_{0}(\lambda)$ of these $\lambda$-models we obtain the right Binder cumulants for the diluted one. Obviously when the mass term is zero we recover the MF result (30) for the pure model.

\section{2 $\quad$ FSS in the diluted model}

The scaling of the singular part of the free energy in the presence of a magnetic field, $h_{0}$, is

$$
f_{\text {sing }}\left(r_{0}, u_{0}, v_{0}, h_{0}, \frac{1}{L}\right)=b^{-4} f_{\text {sing }}\left(r(b), u(b), v(b), h(b), \frac{b}{L}\right),
$$

where we have introduced a new coupling, the system size $L$, which scales trivially with a RG transformation. As usually the magnetic field verifies [9]:

$$
\frac{\mathrm{d} \log h(b)}{\mathrm{d} \log b}=\frac{d}{2}+1-\frac{\gamma_{\phi}(u, v)}{2} .
$$


In the asymptotic regime, the solution of (34) is

$$
h(b)=h_{0} b^{3}(\log b)^{\frac{1}{212}} .
$$

Performing a RG transformation with $b=L$, we keep just one degree of freedom (see ref. [11] for more details). The free energy of this system in the asymptotic regime is

$$
\begin{aligned}
& f\left(r^{\prime}, u^{\prime}, h^{\prime}, L=1\right) \equiv \log \int\left(\prod_{i=1}^{n} \mathrm{~d} \phi_{i}\right) \times \\
& \exp \left\{-\left[\frac{r^{\prime}}{2} \sum_{i=1}^{n} \phi_{i}^{2}-h^{\prime} \sum_{i=1}^{n} \phi_{i}+\frac{u^{\prime}}{4 !}\left(\sum_{i=1}^{n} \phi_{i}^{2}\right)^{2}-\frac{u^{\prime}}{3 !} \sum_{i=1}^{n} \phi_{i}^{4}\right]\right\} .
\end{aligned}
$$

We re-scale the $\phi_{i}$ variables by means of $\phi_{i}^{\prime}=u^{1 / 4} \phi_{i}$. The free energy can be written as

$$
f\left(r^{\prime}, u^{\prime}, h^{\prime}, L=1\right)=\hat{f}\left(\frac{r^{\prime}}{u^{\prime 1 / 2}}, 1, \frac{h^{\prime}}{u^{\prime 1 / 4}}\right),
$$

obtaining finally

$$
f_{\text {sing }}\left(r_{0}, u_{0}, v_{0}, h_{0}, \frac{1}{L}\right)=L^{-4} \hat{f}\left(\frac{r(L)}{u(L)^{1 / 2}}, 1, \frac{h(L)}{u(L)^{1 / 4}}\right) .
$$

We remark that the $u$ variable is a dangerous (marginally) irrelevant variable [12, 13], and we need to do with care all the analytical steps (it is not correct to substitute $u$ for its asymptotically value, $u=0$, because the free energy depends on inverse powers of $u$ ).

As $\hat{f}$ is an analytical function, the $n \rightarrow 0$ limit can be taken in the RHS of (38) substituting $r, h, u$ by their limiting values. For clarity, in the following we will omit the $L$ dependence in the functions $r, u$ and $h$.

To compute the thermodynamical quantities in the critical region one just need to take the appropriate derivatives of $f_{\text {sing. }}$. It will prove convenient to keep in mind eqs. (17) and (33), and the following Taylor expansion (which depends on the relations $r=t+4 K_{4} u$ and the fact that $t(L)=0$ whenever $\left.t_{0}=0\right)$

$$
\begin{aligned}
\left.\partial_{i}^{2} \hat{f}\left(r / u^{1 / 2}, 1,0\right)\right|_{t_{0}=0} & =\partial_{i}^{2} \hat{f}\left(4 K_{4} u^{1 / 2}, 1,0\right) \\
& =\partial_{i}^{2} \hat{f}(0,1,0)+O\left(u^{1 / 2}\right)
\end{aligned}
$$


where $\partial_{i}$ is the partial derivative with respect to the $i$-th argument.

As we are interested in the behavior with the lattice size just at the infinite volume critical temperature, the susceptibility can be written as

$$
\begin{aligned}
\chi & \left.\propto \frac{\partial^{2} f_{\text {sing }}}{\partial h_{0}^{2}}\right|_{h_{0}=t_{0}=0}=\left.L^{-4}\left(\frac{\partial h}{\partial h_{0}}\right)^{2} \frac{\partial^{2}}{\partial h^{2}} \hat{f}\left(r / u^{\frac{1}{2}}, 1, h / u^{\frac{1}{4}}\right)\right|_{h_{0}=t_{0}=0} \\
& \simeq L^{2}(\log L)^{\frac{1}{4}+\frac{1}{106}}
\end{aligned}
$$

The specific heat can be computed analogously

$$
\begin{aligned}
C & \left.\propto \frac{\partial^{2} f_{\text {sing }}}{\partial t_{0}^{2}}\right|_{h_{0}=t_{0}=0}=\left.L^{-4}\left(\frac{\partial r}{\partial t_{0}}\right)^{2} \frac{\partial^{2}}{\partial r^{2}} \hat{f}\left(r / u^{\frac{1}{2}}, 1, h / u^{\frac{1}{4}}\right)\right|_{h_{0}=t_{0}=0} \\
& \simeq(\log L)^{\frac{1}{2}} \mathrm{e}^{-\sqrt{\frac{48}{53}|\log L|}}
\end{aligned}
$$

At zero magnetic field, the correlation length scales as

$$
\left.\xi\left(r_{0}, u_{0}, 1 / L\right)\right|_{t_{0}=0}=\left.L \xi(r, u, 1)\right|_{t_{0}=0}
$$

where $\xi(r, u, 1)$ must be evaluated with the free energy (37). Consequently, the mass squared term is

$$
\left(\left.\xi(r, u, 1)\right|_{t_{0}=0}\right)^{-2}=\left.\frac{r}{u^{1 / 2}}\right|_{t_{0}=0} \propto u^{1 / 2},
$$

and so

$$
\xi\left(r_{0}, u_{0}, 1 / L\right) \propto \frac{L}{u^{1 / 4}} \simeq L(\log L)^{\frac{1}{8}} .
$$

Finally we can also compute the shift of the apparent critical temperature. It can be defined as the temperature where the susceptibility (or specific heat) measured in a finite volume shows a maximum. Using the formula (40) for the susceptibility without imposing the constraint $t_{0}=0$ we obtain

$$
\chi \propto L^{2}(\log L)^{\frac{1}{4}+\frac{1}{106}} \partial_{3}^{2} \hat{f}\left(r / u^{1 / 2}, 1,0\right) .
$$

The maximum of $\chi$ as a function of $L$ and $t$ is not just at $t_{0}=0$, but it is fixed by the condition

$$
r / u^{\frac{1}{2}}=\left(t+4 K_{4} u\right) / u^{\frac{1}{2}}=x_{\max },
$$

i.e. the function $\partial_{3}^{2} \hat{f}(x, 1,0)$ has a maximum at $x=x_{\max }$. 
As $t \propto T_{\mathrm{c}}(\infty)-T_{\mathrm{c}}(L)$, it follows that

$$
T_{\mathrm{c}}(\infty)-T_{\mathrm{c}}(L) \propto L^{-2}(\log L)^{-\frac{1}{4}} \mathrm{e}^{\sqrt{\frac{12}{53} \log L}} .
$$

To finish this section we will report, for completeness, the finite size formulas for the same observables in the pure case [14]:

$$
\begin{aligned}
\xi & \propto L(\log L)^{\frac{1}{4}}, \\
\chi & \propto L^{2}(\log L)^{\frac{1}{2}}, \\
C & \propto(\log L)^{\frac{1}{3}} \\
T_{\mathrm{c}}(\infty)-T_{\mathrm{c}}(L) & \propto L^{-2}(\log L)^{-\frac{1}{6}} .
\end{aligned}
$$

The latter expressions can also be obtained with the method described above.

\section{Numerical Methods}

The choice of the Monte Carlo (MC) update algorithm should be carefully considered. The Wolff single cluster method [15] is the best choice for the pure model. However in the diluted case, small isolated clusters of spins are very likely to appear. Those clusters are scarcely visited by the Wolff method. Therefore, we have complemented the updating method with a Metropolis sweep per measure. We have checked that this algorithm thermalizes appropriately the configurations for $p \geq 0.5$, by comparing the numerical results from cold and hot starts. However, for $p \leq 0.4$, equilibration by this method becomes really hard to achieve. This is due to the presence of intermediate size clusters almost isolated from the percolating one. We have then turned to the Swendsen-Wang (SW) [16] algorithm which guarantees that all-sized spin-clusters are considered. In this way, we find complete agreement between hot and cold starts. We have also compared the results of a SW and a single-cluster simulation at $p=0.5$ on our largest lattice, finding compatible results. However, to get a statistically-independent new configuration takes significantly longer (in CPU time) with the SW algorithm.

For every observable, one first averages in the sample, then averages between different samples. Therefore, a crucial point is how long each sample simulation should be. Assuming full statistical independence between different measures (quite possible with a cluster method), and also between measures taken in different samples, the variance of such a mean is 


$$
\sigma_{T}^{2}=\frac{1}{N_{S}}\left(\sigma_{S}^{2}+\frac{\sigma_{I}^{2}}{N_{I}}\right)
$$

where $N_{S}$ is the number of samples generated and $N_{I}$ is the number of measures in each Ising-model simulation. The variance between samples of the thermal average of our observable is $\sigma_{S}^{2}$ and, finally, $\sigma_{I}^{2}$ is the average of the variances in each sample. On the other hand, the computational effort is roughly proportional to $N_{S} N_{I}$, as the computer usually spends a fixed fraction of the time measuring. It is then clear that the optimum value of $N_{I}$ cannot be much bigger that $\sigma_{I}^{2} / \sigma_{S}^{2}$. One could even be tempted to measure just once by sample. However, we shall come back to this point when discussing re-weighting methods.

\subsection{Derivatives and Re-weighting Methods}

The critical curve slope (see fig. (1) changes quite abruptly. It is nearly vertical for large $p$ and almost horizontal close to the percolation threshold. It is therefore wise to choose a re-weighting method such that we may extrapolate to different $\beta$ values close to the pure model, but to different $p$ values at very strong dilution. Let us then comment both extrapolation methods separately.

\subsection{1 $\beta$-extrapolation}

The energy measures allow the calculation of $\beta$-derivatives of observables, and the use of the standard re-weighting methods, before the sample-average is performed. However, an important point should be made now, so let us recall how are they calculated:

$$
\begin{gathered}
\partial_{\beta} \overline{\langle\mathcal{O}\rangle}=\overline{\partial_{\beta}\langle\mathcal{O}\rangle}=\overline{\langle\mathcal{O E}-\langle\mathcal{O}\rangle\langle\mathcal{E}\rangle\rangle}, \\
\langle\mathcal{O}\rangle(\beta+\Delta \beta)=\left\langle\mathcal{O} \mathrm{e}^{\Delta \beta \mathcal{E}}\right\rangle /\left\langle\mathrm{e}^{\Delta \beta \mathcal{E}}\right\rangle .
\end{gathered}
$$

It is clear that both expressions are biased. For instance, the expectation value of eq. (50), when the averages are calculated with $N_{I}$ measures, is really

$$
\overline{\left(1-\frac{2 \tau}{N_{I}}\right) \partial_{\beta}\langle\mathcal{O}\rangle},
$$




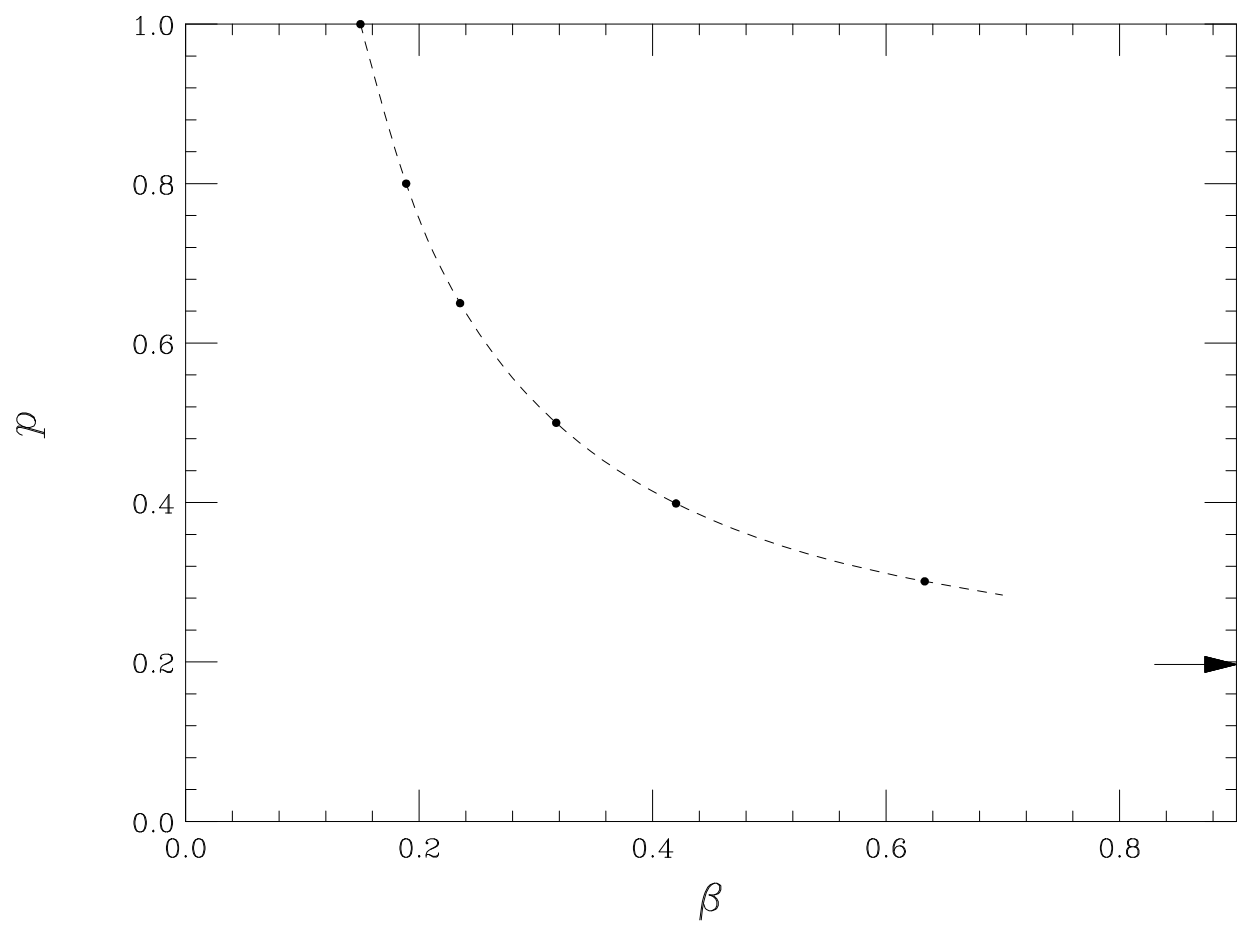

Figure 1: Phase diagram of the four dimensional site-diluted Ising model. The points correspond to the simulated values, and the arrow indicates the percolation limit.

$\tau$ being the integrated autocorrelation time [17], which depends on the sample. The bias for eq. (51) is also of order $2 \tau / N_{I}$, but terms of higher order in $1 / N_{I}$ are to be expected.

These biases are immaterial for usual MC investigations, as the statistical error decreases with the square root of the number of measurements. However, in our case the bias is of order $1 / N_{I}$, while the statistical error is of order $1 / \sqrt{N_{S}}$, which are similar in our simulations!

The cure for this is to introduce unbiased estimators. A possible method would be to measure in completely independent samples, ensuring $2 \tau=1$ for every sample. In this case the unbiased estimator is constructed multiplying by $1 /\left(1-1 / N_{I}\right)$ the $\beta$-derivatives and by other more complex functions the extrapolated observables. However, this would be too expensive from the computational point of view. The solution we find is to work with $\tau \gtrsim 1$, 
repeat the calculations with different values of $N_{I}$ and extrapolate $N_{I} \rightarrow \infty$.

Specifically, in each sample, we calculate the derivative with the full MC history, obtaining a number $y_{1}$, the bias being proportional to $2 \tau / N_{I}$. We then consider two contiguous halves of the MC history, repeat the calculation for each one and average the final result. This value, $y_{2}$, has a bias that goes as $4 \tau / N_{I}$. The next term, $y_{3}$, is obtained with four quarters with a bias proportional to $8 \tau / N_{I}$.

The linear extrapolation is

$$
y_{L}=2 y_{1}-y_{2}
$$

and the quadratic one

$$
y_{Q}=\frac{8}{3} y_{1}-2 y_{2}+\frac{1}{3} y_{3}
$$

We then average $y_{L}$ and $y_{Q}$ for all the samples, checking that the difference is negligible compared with the statistical error. In fact, we have found that the slope in the $\left(y, 1 / N_{I}\right)$ plane is a very clean and easy measure of $\tau$, which we have used to achieve statistical independence between different measures. See fig. 2 for an example.

We proceed analogously with the extrapolations of observables or their derivatives.

Another approach to eliminate the biases is to split the measures in statistically independent sets and multiply the average of some operator in the first set by the average of another operator in the second set. In some cases (for instance derivatives extrapolated at different couplings) it could be necessary to work with more than two independent sets. As in practice one

have contiguous MC measures, the statistical independence of the measures becomes involved when splitting in several sets.

The comparison between the quadratic extrapolation and the linear one makes it possible to monitor this short $M C$ history effects. In some cases due to the necessity of a large extrapolation in the coupling, we have found differences between $y_{L}$ and $y_{Q}$ around one half of the statistical error; then we have repeated the simulation at a nearer point.

\subsection{2 p-extrapolation}

In addition to the standard $\beta$-extrapolation [18], it is also possible to extrapolate the mean values obtained at a given dilution probability, $p$, to a close 


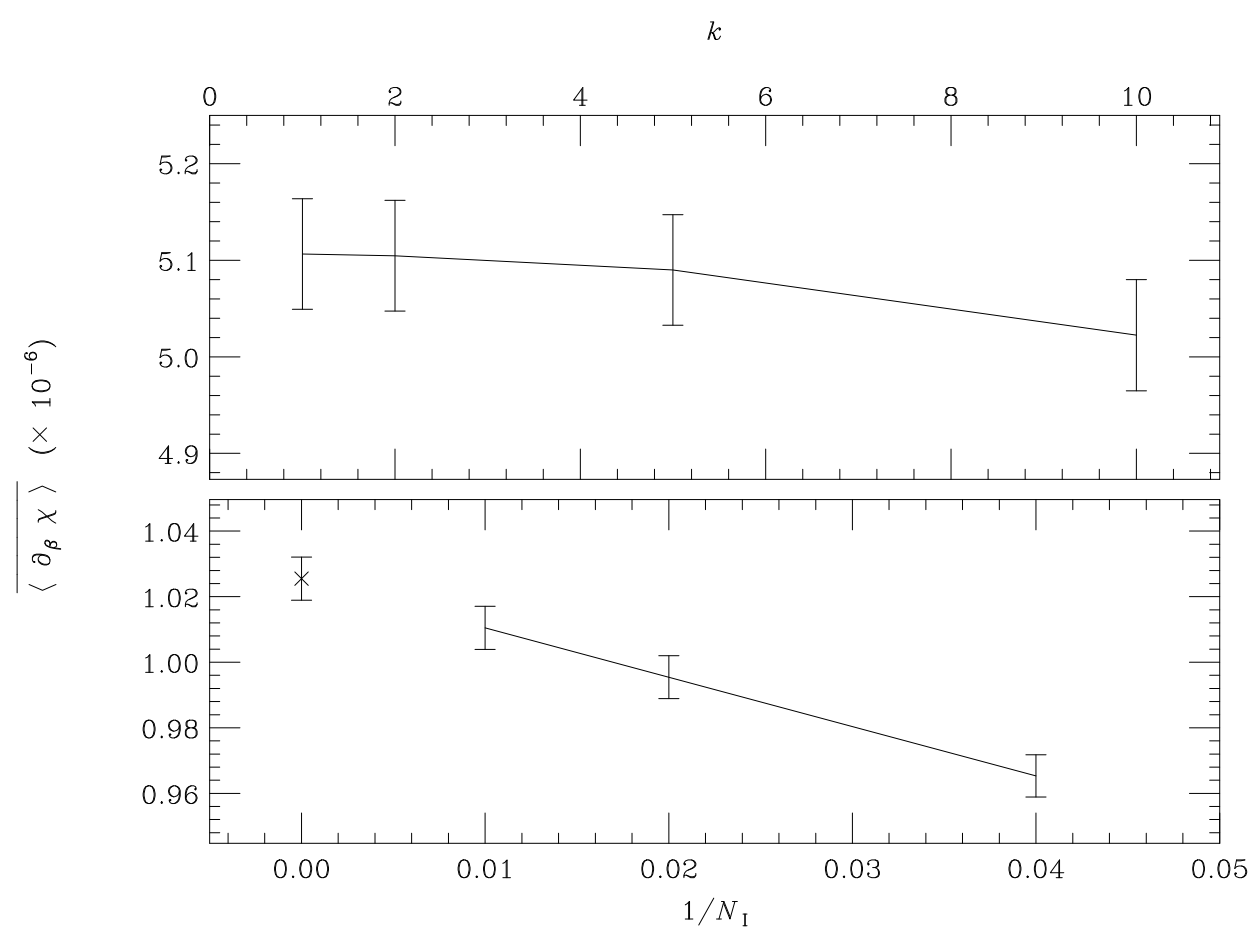

Figure 2: In the upper part we show the sample-averaged $y_{1}$ value for $\partial_{\beta} \chi$, taking one out of each $k$ measures, in a $L=48$ lattice at $p=0.5$, with $\tau \approx 8$. As both $\tau$ and $N_{I}$ get divided by $k$, we obtain an stable value until $k=10$. In the lower part, we plot the sample-averaged $y_{1}, y_{2}$ and $y_{3}$ values, as a function of the inverse number of MC measures, in a $L=32$ lattice, at $p=0.5$, with $\tau \approx 0.8$. The linear behavior is apparent.

one $p^{\prime}$ (see [19]). Let us simply recall that the probability of finding a occupation number $q$, when filling sites with probability $p$, is binomial. Therefore the re-weighting is calculated from a set of $N_{S}$ mean values of an observable $\mathcal{O}$ and the actual density of the configuration $\left\{\left(\langle\mathcal{O}\rangle_{i}(\beta), q_{i}\right)\right\}$ :

$$
\overline{\langle\mathcal{O}\rangle}\left(p^{\prime}, \beta\right)=\frac{1}{N_{S}} \sum_{i}^{N_{S}}\left(\frac{p^{\prime}}{p}\right)^{q_{i} V}\left(\frac{1-p^{\prime}}{1-p}\right)^{\left(1-q_{i}\right) V}\langle\mathcal{O}\rangle_{i}(\beta)
$$

The visible region is of course constrained to the variance of the binomial distribution $p(1-p) / V$. Fortunately, it has been enough for us.

Using equation (55) $p$-derivatives of observables can also be computed, 
but statistical errors are about eight times bigger than for $\beta$-derivatives. Therefore, our choice is to study $p$-extrapolated $\beta$-derivatives, where all the above comments for the bias in the derivative are in order, but the reweighting is unbiased.

\subsection{Numerical FSS techniques}

As already stated in the introduction, we consider the possibility of finding a non-Gaussian fixed point in four dimensions, where hyperscaling relations were fulfilled. In such a case, the usual FSS ansatz is expected to hold. However, the PRG analysis rather suggests the presence of logarithmic corrections to the Gaussian behavior. Therefore, we should also consider the modifications in the FSS ansatz induced by the logarithmic corrections. In this section, we shall first remind how critical exponents are measured (see refs. [4, 19] for similar calculations), then we shall show how to deal with logarithmic corrections.

When hyperscaling holds, a very accurate way of measuring critical exponents involves a form of the FSS ansatz where everything is directly measurable on a lattice:

$$
O(L, \beta, p)=L^{x_{O} / \nu}\left(F_{O}(\xi(L, \beta, p) / L)+O\left(L^{-\omega}\right)\right),
$$

where a critical behavior $t^{-x_{O}}$ is expected for the operator $O, \omega$ is the universal scaling-corrections exponent, and $F_{O}$ is a (smooth) scaling function. Notice that terms of order $\xi_{L=\infty}^{-\omega}$ are dropped from eq. (56), so we are deep

within the scaling region. From a Renormalization Group point of view, $\omega$ corresponds to the leading irrelevant operator.

In order to calculate the critical exponents, we study the quotient of $O(s L)$ and $O(L)$, defined as

$$
Q_{O}=O(s L, \beta, p) / O(L, \beta, p) .
$$

Measuring at a value of the couplings where the quotient for the correlationlength is $s$ the scaling function may be eliminated and we obtain:

$$
\left.Q_{O}\right|_{Q_{\xi}=s}=s^{x_{O} / \nu}+O\left(L^{-\omega}\right) .
$$

If there are logarithmic corrections to hyperscaling, the critical behavior of the operator $O$ is modified to

$$
O\left(L, \beta_{c}, p_{c}\right) \propto L^{x_{O} / \nu}(\log L)^{\delta_{O}} .
$$


We remark that the scaling variable is again $\xi(L, \beta, p) / L$. This is clear from formulas (42), (43) and (38): the scaling variable is $r / u^{1 / 2}$ that is equal to $(\xi / L)^{2}$, and so one readily obtains that the scaling variable is $\xi / L$ without logarithmic corrections.

For the susceptibility exponent we find from eq. (40) after some algebra

$$
\frac{x_{\chi}}{\nu}=\frac{\left.\log Q_{\chi}\right|_{Q_{\xi}=s}}{\log s}+O\left(\frac{1}{\log L}\right) .
$$

Thus, the corrections to the $\eta$ exponent are proportional to $1 / \log L$ as in the pure case.

To estimate the logarithmic correction to the critical behavior of the $\beta$ derivative of the correlation length we start from eqs. (42) and (43) and get

$$
\xi=\xi\left(r_{0}, u_{0}, 1 / L\right)=L \frac{u^{1 / 4}}{r^{1 / 2}} .
$$

Taking the $t_{0}$ derivative and using eqs. (17) we obtain

$$
\partial_{\beta} \xi \propto L^{3}(\log L)^{1 / 4}\left(\frac{\xi}{L}\right)^{3} \exp \left[-2 \sqrt{\frac{3 \log L}{53}}\right] .
$$

It is easy to check that

$$
\frac{x_{\partial_{\beta} \xi}}{\nu}=\frac{\left.\log Q_{\partial_{\beta} \xi}\right|_{Q_{\xi}=s}}{\log s}+O\left(\frac{1}{\sqrt{\log L}}\right)
$$

where the correction $O(1 / \sqrt{\log L})$ arises from the exponential term in eq. (62).

For the lattice sizes simulated, we expect some dependence on the dilution in the coefficient of the $1 / \sqrt{\log L}$ term. In the initial regime the corrections are proportional to $1 / \log L$ (the pure model). Until the system forget the initial conditions (in particular the dependence on the dilution) the coefficient of the $1 / \sqrt{\log L}$ term could change.

\section{$5 \quad$ Numerical Results}

The lattice sizes that we have studied have been $L=8,12,16,24$ and 32 . We have generated $N_{S}=10,000$ samples, for each lattice size, at dilution values $p=0.8,0.65,0.5,0.4,0.3$. In each sample, we measure $N_{I}=100$ times 
after equilibration. The number of clusters traced (or SW updates) between measures have been chosen to yield $2 \tau \approx 1$ (see eq. (52)). The pure $(p=1)$ model has also been studied for $L=8,12,16,24,32,48$ and 64 , as a contrast of the disorder-induced effects.

We shall present our numerical results in two steps. First we shall consider the conventional FSS analysis (i.e. assuming hyperscaling), finding that the percolation scenario is extremely unlikely.

After that, we shall look for hyperscaling violations in the data. We shall find that they can be measured, and are indeed of the same order as predicted by PRG.

\section{$5.1 \quad$ Assuming Hyperscaling}

To measure the critical exponents, we use the so-called quotients method, which allows for a great statistical accuracy [4, 19]. The starting point is eq. (58). We have first approximately located the point where

$$
\frac{\xi(2 L, \beta, p)}{2 L}=\frac{\xi(L, \beta, p)}{L},
$$

then we have used re-weighting techniques to fine-tune the condition (64). For $p=1.0,0.8,0.65$ and 0.5 , we have used, $\beta$-extrapolation. Therefore, the critical $p$ is fixed, $\beta$ being the tunable parameter. For lower dilution values, we have rather used $p$-extrapolation, so we have first approximately located the $\beta$ value, for which condition (64) holds at $p \simeq 0.3,0.4$. Next we fix $\beta$, fine-tuning $p$ afterwards, so the critical values differ from $p=0.3,0.4$ by an amount of less than 1\%. However, in tables and figures, we shall refer to them as $p=0.4$ and 0.3 for brevity.

Eq.(58) applied to the operators $\partial_{\beta} \xi$ and $\chi$, yields respectively the exponents $1+1 / \nu$ and $2-\eta$. The numerical results are shown in tables 1 and 2. For the $\nu$ exponent we find, instead of a stable value, a monotonically decreasing one. For $\eta$, such an evolution with growing $L$ is found, but it is clearly weaker. Therefore, an infinite volume extrapolation is called for. If hyperscaling holds, we expect finite-volume scaling-corrections proportional to $L^{-\omega}$. As $\omega=1.13(10)$ in the percolation [19], in the last row of both tables 1 and 2 we include an infinite-volume extrapolation with $\omega=1$. This fit is shown in figure 3 .

It is clear that the percolation scenario, $\nu=0.686(2)$ and $\eta=-0.094(3)$ [19], can be ruled out. Moreover, the possibility of a different fixed point 


\begin{tabular}{|r|c|c|c|c|c|c|}
\hline$L$ & $p=1.0$ & $p=0.8$ & $p=0.65$ & $p=0.5$ & $p \simeq 0.4$ & $p \simeq 0.3$ \\
\hline \hline 8 & $0.5119(9)$ & $0.5175(11)$ & $0.5308(13)$ & $0.5482(16)$ & $0.5604(15)$ & $0.5700(26)$ \\
\hline 12 & $0.5074(18)$ & $0.5154(11)$ & $0.5270(13)$ & $0.5428(19)$ & $0.5532(19)$ & $0.5647(22)$ \\
\hline 16 & $0.5066(10)$ & $0.5142(13)$ & $0.5251(12)$ & $0.5412(19)$ & $0.5478(18)$ & $0.5583(26)$ \\
\hline 24 & $0.5067(18)$ & & & & & \\
\hline 32 & $0.5039(17)$ & & & & & \\
\hline$\infty$ & $0.5019(14)$ & $0.5110(25)$ & $0.5194(26)$ & $0.534(4)$ & $0.536(4)$ & $0.549(5)$ \\
\hline
\end{tabular}

Table 1: The $\nu$ exponent for $(L, 2 L)$ pairs at the different dilutions.

\begin{tabular}{|r|c|c|c|c|c|c|}
\hline$L$ & $p=1.0$ & $p=0.8$ & $p=0.65$ & $p=0.5$ & $p \simeq 0.4$ & $p \simeq 0.3$ \\
\hline \hline 8 & $-0.02644(14)$ & $-0.0161(12)$ & $-0.0124(11)$ & $-0.0055(19)$ & $0.002(8)$ & $-0.003(4)$ \\
\hline 12 & $-0.02132(26)$ & $-0.0138(12)$ & $-0.0089(13)$ & $-0.0051(17)$ & $0.000(9)$ & $-0.0043(21)$ \\
\hline 16 & $-0.01656(12)$ & $-0.0130(12)$ & $-0.0053(10)$ & $-0.0073(17)$ & $0.000(11)$ & $-0.006(7)$ \\
\hline 24 & $-0.01259(29)$ & & & & & \\
\hline 32 & $-0.01445(18)$ & & & & & \\
\hline$\infty$ & $-0.0085(18)$ & $-0.0097(25)$ & $0.0013(22)$ & $-0.008(4)$ & $-0.002(20)$ & $-0.007(9)$ \\
\hline
\end{tabular}

Table 2: The $\eta$ exponent for $(L, 2 L)$ pairs at different dilutions.

neither Gaussian nor with the percolation critical exponents requires a fairly exotic FSS behavior. We do not find this possibility likely.

At this point, one could claim that this model presents weak universality, as recently proposed in the two dimensional version of the model [20]. That is, the exponent $\eta$ is constant over the critical line, while $\nu$ is continuously varying. However, a much less spectacular, but more likely interpretation will be given in the next subsection.

\subsection{The quest for logarithms}

As PRG predicts logarithmic corrections to the MF behavior, we should expect scaling-corrections of order $1 / \log L$ (for the $\eta$ and $\nu$ exponents in the pure model and only for the $\eta$ exponent in the diluted case) and $1 / \sqrt{\log L}$ (for the $\nu$ exponent in the diluted one): both are of the same order for the lattices that we can afford!. In figures 1 and 5 we show that the deviation from MF can indeed be accounted for by logarithmic corrections. We have found similar results in two dimensions [21]. While finishing these papers, the same conclusion has been independently drawn in a transfer-matrix study of 


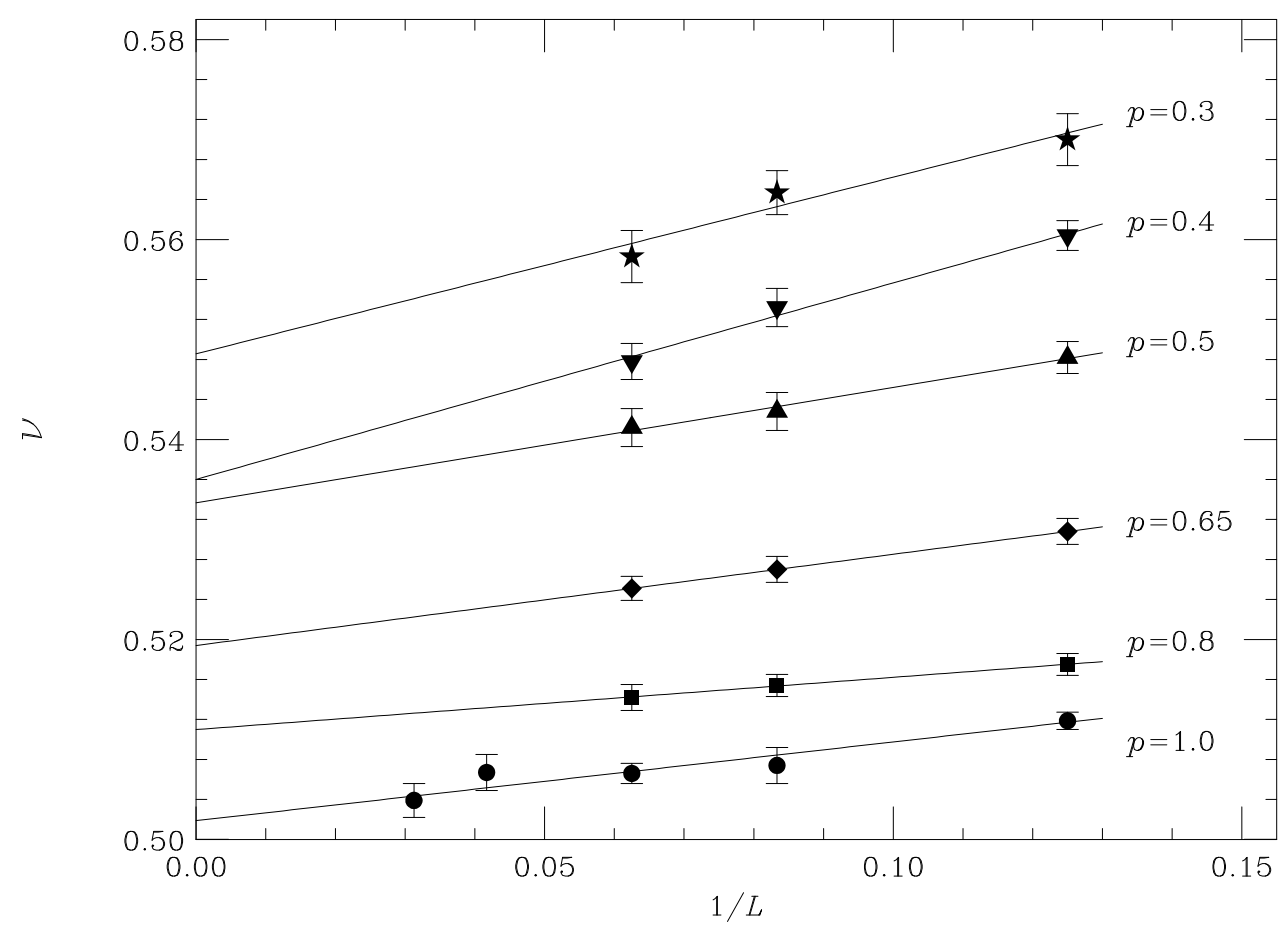

Figure 3: Exponent $\nu$ for different lattice pairs of type $(L, 2 L)$. The lines correspond to linear fits.

the bond-diluted two dimensional model [22].

As we have seen, hyperscaling does not seem to hold. Indeed, the PRG predict logarithmic violations. In this section, we try to identify them by starting from a naive point of view. That is, we shall first locate the critical point as if the usual FSSA were correct, and then we shall study there the scaling of physical quantities, looking for deviations from the pure-power law.

A very accurate way of computing $\beta_{\mathrm{c}}$ (or $p_{\mathrm{c}}$ ) is to fix a value of $g_{4}$, measuring in what $\beta$ the function $g_{4}(L, \beta)$ is equal to the fixed previous value [24]. In absence of logarithmic corrections one expects that the shift in $\beta$ behaves as $1 / L^{2}$. In figure 6 we plot these quantities for three values of $g_{4}$ at each $p$, while the results of a quadratic fit, for the infinite volume critical couplings are found in table 3. We point out that a logarithmic correction as the one computed in ref. [14] (as eq. (49) shows) for the pure model or eq. (47) for the diluted one, does not change the fit results. 


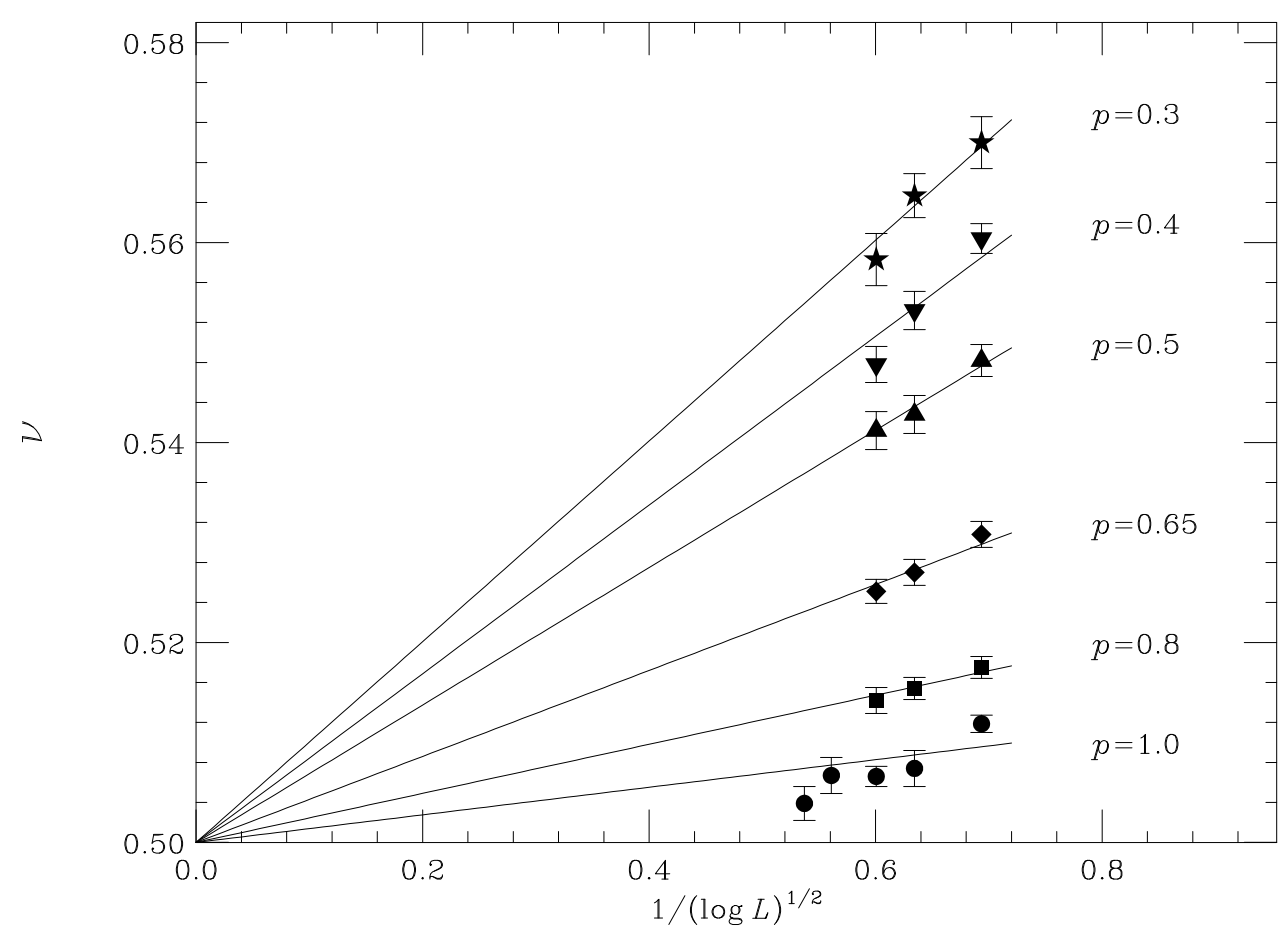

Figure 4: The $\nu$ exponent obtained from $(L, 2 L)$ pairs. The solid lines are linear fits constrained to be $\nu=0.5$ in the $L \rightarrow \infty$ limit.

In order to minimize the systematic errors, those values have been obtained choosing a $g_{4}$ such that the linear coefficient in $1 / L^{2}$ vanishes. We have also computed the extrapolations for a wide range of $g_{4}$ values to check the amplitude of the change. We observe that, within one standard deviation in the extrapolated value, $g_{4}$ can be changed in the interval $[0.3,0.8]$ in the best case $(p=0.8)$ and in the interval $[0.4,0.6]$ in the worst one $(p=0.5)$. Although we think that systematic errors from this source are negligible, a more conservative attitude could be to duplicate the statistical error bars in table 3 .

Now, we can check hyperscaling violations of the form:

$$
\xi\left(L, \beta_{c}\right) \propto L(\log L)^{\delta_{\xi}} .
$$

In figure 7, we plot $\log (\xi / L)$ as a function of $\log (\log L)$, the slope being directly $\delta_{\xi}$. We can see that a good linear behavior is obtained. The fitted $\delta_{\xi^{-}}$ 

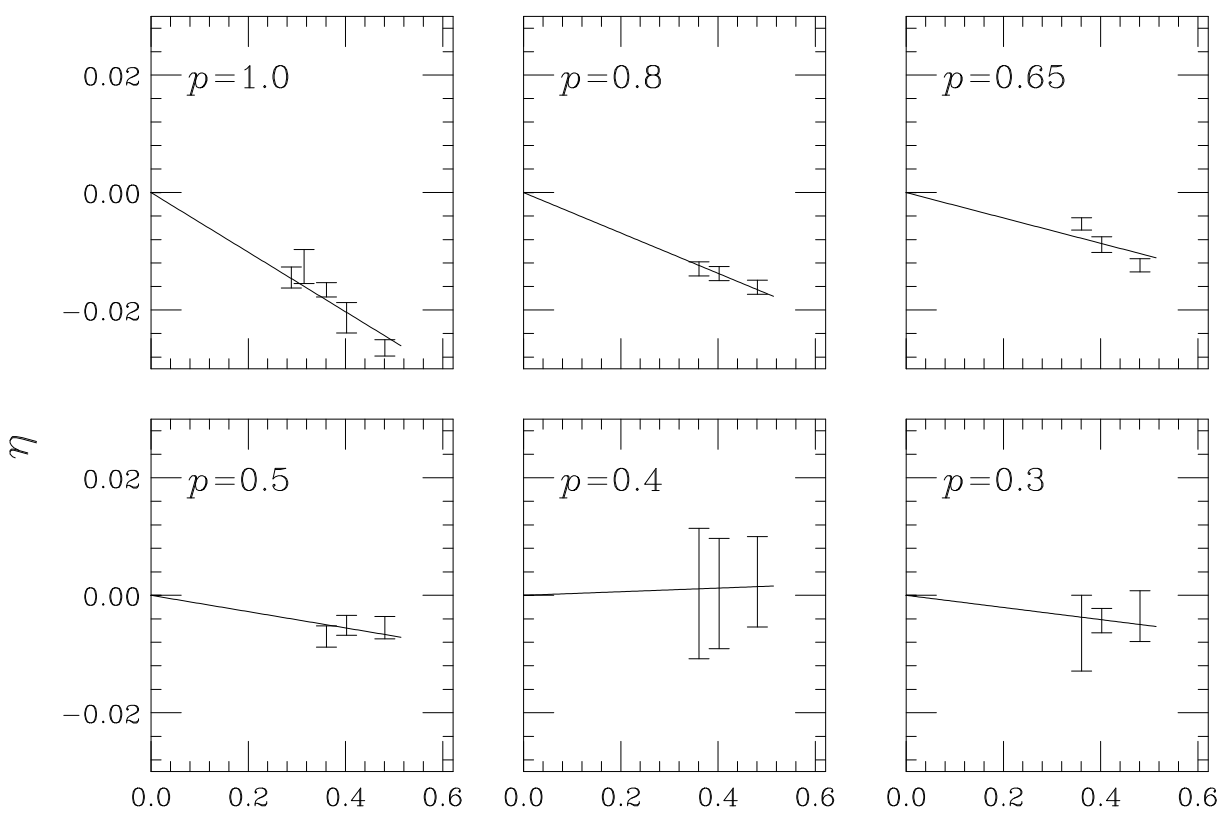

\section{$1 / \log L$}

Figure 5: The $\eta$ exponent for different lattice sizes. The fit is enforced to yield $\eta=0$ for $L \rightarrow \infty$.

values are reasonably close to the theoretical prediction, but a growing trend with the dilution is self-evident. A naive (wrong) explanation is that this is an effect coming from the vicinity of the percolation critical point. Indeed, we can repeat the previous fit in the pure percolation for similarly-sized lattices. Fitting from $L=8$ we obtain $\delta_{\xi}=0.0927(9)$ with $\chi^{2} /$ d.o.f. $=97.9$ and from $L=16$, the fit parameters are $\delta_{\xi}=0.066(2)$ with $\chi^{2} /$ d.o.f. $=6.90$. So a linear behavior is ruled out for this model. However, this is not surprising, as hyperscaling is known to hold for percolation in four dimensions.

Another interesting observable is the specific heat, which for the pure model is expected to diverge as $C \propto(\log L)^{1 / 3}$, while in the diluted case it is expected to remain bounded. Both predictions can be tested. The fitted 

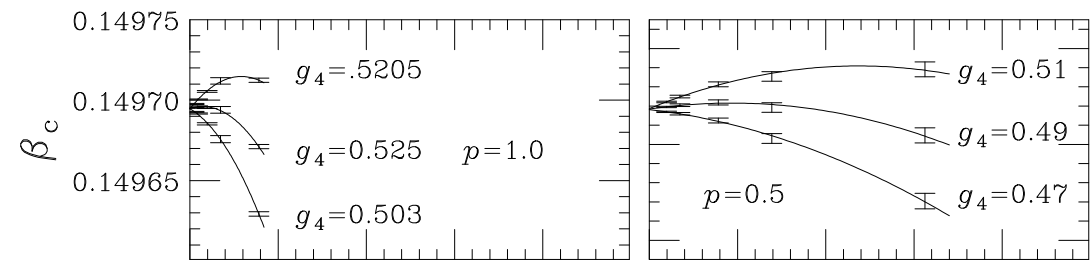

0.318

$0.317 \boldsymbol{0}^{0}$

0.316
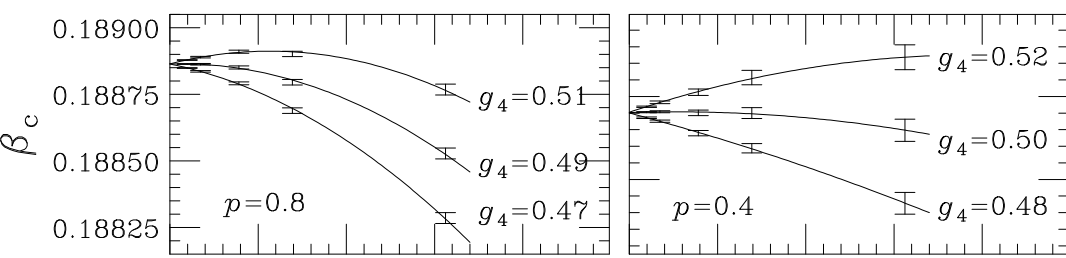

0.399

$2^{0}$ 0.398
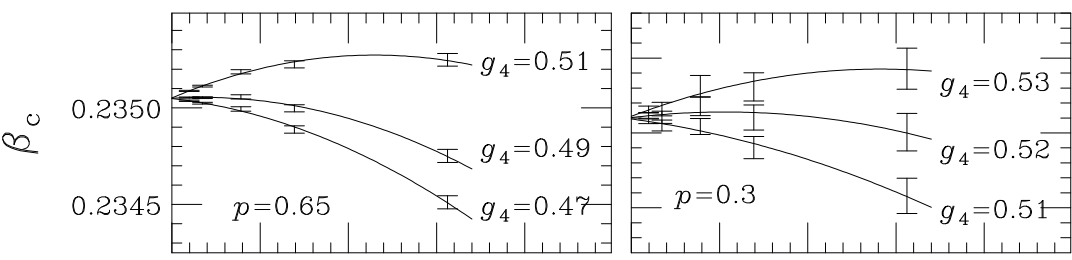

0.3015

0.30102

0.3005

\section{$1 / L^{2}$}

Figure 6: Computation of $\beta_{\mathrm{c}}$ or $p_{\mathrm{c}}$ looking at fixed values of $g_{4}$ for the different values of the dilution. The lines are quadratic fits.

values for the logarithmic divergence exponent, $\delta_{C}$, are

$$
\begin{array}{ll}
p=1.0: & \delta_{C}=0.399(4+22), \\
p=0.8: & \delta_{C}=0.304(7+13), \\
p=0.65: & \delta_{C}=0.184(8+15), \\
p=0.5: & \delta_{C}=0.095(6+9), \\
p=0.4: & \delta_{C}=0.084(5+6), \\
p=0.3: & \delta_{C}=0.073(8+7) .
\end{array}
$$

On the values of the Binder cumulants at the infinite volume critical temperature we can see directly the (logarithmic) corrections to the scaling . In the pure case we have obtained $g_{4} \simeq 0.51$ for the largest lattice at the critical point: we remark that the asymptotic value for the pure model is

${ }^{1}$ Do not confuse with the multiplicative logarithmic corrections to the critical law that we have studied above. 


\begin{tabular}{|l|l|}
\hline \multicolumn{1}{|c|}{$p_{\mathrm{c}}$} & \multicolumn{1}{c|}{$\beta_{\mathrm{c}}$} \\
\hline 1.0 & $0.149695(1)$ \\
\hline 0.8 & $0.188864(3)$ \\
\hline 0.65 & $0.235049(8)$ \\
\hline 0.50 & $0.317368(19)$ \\
\hline $0.398806(18)$ & 0.42 \\
\hline $0.30110(4)$ & 0.633 \\
\hline
\end{tabular}

Table 3: Critical couplings as obtained from a quadratic fit of $\beta_{c}\left(L, g_{4}\right)$. The error bars are purely statistical.

$g_{4} \simeq 0.406$. It is possible to show using [25] that the leading correction to scaling term for $g_{4}$ goes as $1 / \log L$ in both the pure model and the diluted one. Our pure $g_{4}$-data are compatible with a limiting value of 0.406 modified by $1 / \log L$ corrections.

In the diluted model, we have obtained data for the $g_{4}$ cumulant around 0.5 (see fig. 6) which is quite larger than the predicted value $g_{4} \simeq 0.32$ (see eq. (29)). Again, this numerical discrepancy can be understood taking into account corrections like $1 / \log L$. The same comments hold for $g_{2}$.

In figure 8 we have shown $g_{2}$ against $g_{4}$ for some values of the dilution. We have also plotted the theoretical value for the pair $\left(g_{2}, g_{4}\right)$. This figure must be interpreted having in mind the previously cited logarithmic corrections to the scaling. It is clear that the complete numerical characterization of these cumulants needs further numerical work.

Finally we have studied the probability distribution of the observable $\mathcal{M}^{2}$ (i.e. we collect the histogram of the values of $\mathcal{M}^{2}$ at every measure independently of the sample). We have found that for large values of $\mathcal{M}^{2}$ the data follow a law $P\left(\mathcal{M}^{2}\right) \propto \exp \left(-c(L)\left(\mathcal{M}^{2}\right)^{2}\right)$. Using the results of section (3.1) it is possible to show that the theoretical prediction for the probability distribution is a Gaussian with $c(L) \propto u(L) L^{4}$. The coefficient, $c(L)$, computed numerically follows very well a law $L^{4} / \sqrt{\log L}$ in perfect agreement with the theoretical prediction. 

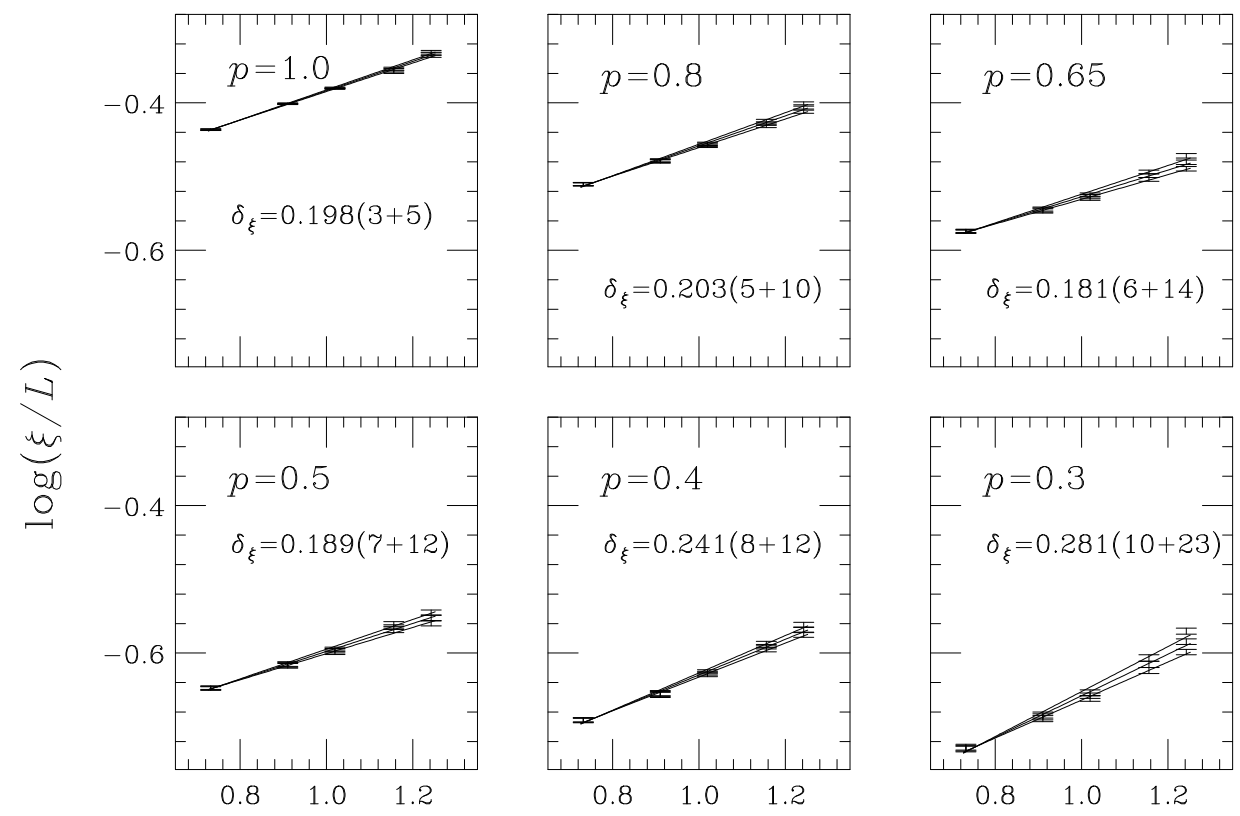

$\log (\log L)$

Figure 7: $\log (\xi / L)$ in the infinite volume critical point, as a function of $\log (\log L)$, for several dilutions. The fitted $\delta_{\xi}$ values are also displayed. The first term in the error is statistical, while the second corresponds to the error in the critical coupling. The three lines correspond to data at the critical point and one standard deviation apart at each side.

\section{Conclusions}

We have performed a Monte Carlo simulation of the site-diluted Ising model in four dimensions, for several values of the dilution, and in a wide range of lattice sizes. The use of a finite-size scaling analysis allows us to consider big lattices just at the critical point. To gain accuracy we have repeated the simulations for many different hole configurations.

As a first stage, we have measured with great precision the critical exponents under the hypothesis of hyperscaling. The value we obtain for the $\nu$ exponent changes along the critical line, ruling out the possibility of a single non-Gaussian fixed point. 


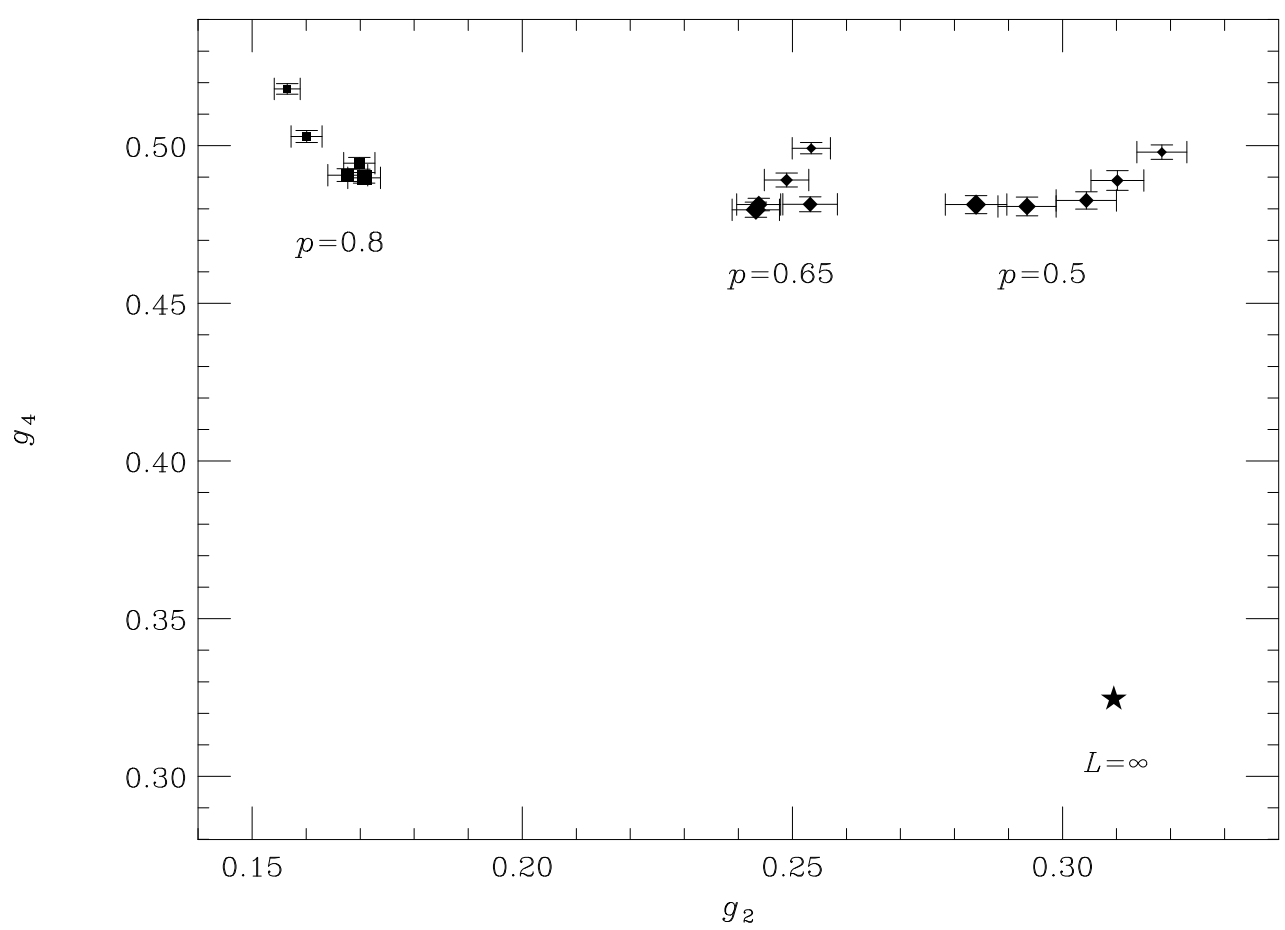

Figure 8: $g_{4}$ versus $g_{2}$ for three values of the dilution. The symbol sizes increase with the lattice size $(L=8,12,16,24,32)$. The PRG prediction is also plotted.

Using Perturbative Renormalization Group techniques, we have computed the scaling formulas for the diluted model, obtaining specific logarithmic corrections to the Gaussian behavior.

We have re-analyzed our numerical data finding that they agree with a Gaussian scenario with logarithmic corrections to hyperscaling. The pure model has also been considered and its corresponding scaling formulas, checked.

Although the nature of the logarithmic corrections hardly allows to perform precise fits to the predicted functional forms, we have found a reasonable agreement. 


\section{Acknowledgments}

We thank to the CICyT (contracts AEN94-0218, AEN96-1634) for partial financial support, specially for the use of dedicated Pentium Pro machines where we have carried out the simulations. JJRL is granted by EC HMC (ERBFMBICT950429). 


\section{References}

[1] A. B. Harris, J. Phys. C7 (1974) 1671.

[2] G. Parisi and J. J. Ruiz-Lorenzo, J. Phys. A: Math. Gen. 28 (1995) L395.

[3] F. Cooper, B. Freedman and D. Preston, Nucl. Phys. B210 (1989) 210.

[4] H. G. Ballesteros, L.A. Fernández, V. Martín-Mayor, and A. Muñoz Sudupe, Phys. Lett. B378 (1996) 207; Phys. Lett. B387 (1996) 125; Nucl. Phys. B 483 (1997) 707.

[5] E. Brèzin, J. Physique 43 (1982) 15.

[6] G. Parisi, Field Theory, Disorder and Simulations (World Scientific, Singapore 1994).

[7] A. Aharony, Phys. Rev. B 13 (1976) 2092.

[8] J. J. Ruiz-Lorenzo. In preparation.

[9] M. Le Bellac, Quantum and Statistical Field Theory (Oxford Science Publications 1991).

[10] J. Zinn-Justin, Quantum Field Theory and Critical Phenomena (Oxford Science Publications 1990).

[11] E. Luijten and W. J. Blöte, Phys. Rev. Lett. 76, 1557 (1996); Erratum 76 (1996) 3662.

[12] A. C. D. van Enter, R. Fernández and A. D. Sokal, J. Stat. Phys.72 (1994) 879.

[13] M. E. Fisher, in Renormalization Group in Critical Phenomena and Quantum Field Theory: Proceedings of a conference. Temple University ( Philadelphia, 1974).

[14] R. Kenna, C.B. Lang, Phys. Lett. B264 (1991) 396; Nucl. Phys. B393 (1993) 461.

[15] U. Wolff, Phys. Rev. Lett. 62 (1989) 3834. 
[16] R. H. Swendsen and J. S. Wang, Phys. Rev. Lett. 58 (1987) 86.

[17] A. D. Sokal, Bosonic Algorithms in Quantum Fields on the Computer. Advanced Series on Direction in High Energy Physics Vol. 11, M. Creutz Editor. World Scientific, Singapore 1992.

[18] M. Falcioni, E. Marinari, M. L. Paciello, G. Parisi and B. Taglienti, Phys. Lett. 108 (1982) 331; A. M. Ferrenberg and R. H. Swendsen, Phys. Rev. Lett. 61 (1988) 2635.

[19] H.G. Ballesteros, L.A. Fernandez, V. Martin-Mayor, A. Munoz Sudupe, G. Parisi, J.J. Ruiz-Lorenzo, Phys. Lett. B400 (1997) 346.

[20] J.K. Kim and A. Patrascioiu, Phys. Rev. Lett 72 (1994) 2785; Phys. Rev. B49 (1994) 15764.

[21] H.G. Ballesteros, L.A. Fernández, V. Martín-Mayor, A. Muñoz Sudupe, G. Parisi, J.J. Ruiz-Lorenzo, cond-mat/9707.

[22] F. D. A. Aarão Reis, S. L. A. de Queiroz and R. R. dos Santos, cond-mat 9706202 .

[23] K. Binder, Z. Phys. B43 (1981) 119.

[24] G. Parisi and J. J. Ruiz-Lorenzo, Phys. Rev. B54 (1996) 3698.

[25] D. J. Amit, Field Theory, the Renormalization Group, and Critical Phenomena (World Scientific, Singapore 1984) (Revised second edition). 